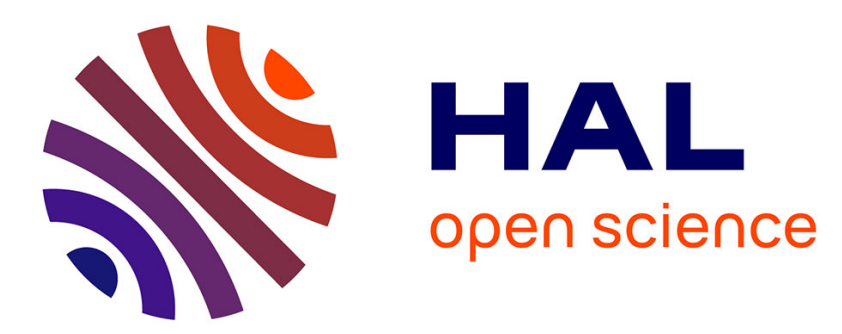

\title{
Experimental characterization of ID-Hall, a double stage Hall thruster with an inductive ionization stage
}

A. Martín Ortega, A. Guglielmi, F. Gaboriau, C. Boniface, Jean-Pierre Boeuf

\section{To cite this version:}

A. Martín Ortega, A. Guglielmi, F. Gaboriau, C. Boniface, Jean-Pierre Boeuf. Experimental characterization of ID-Hall, a double stage Hall thruster with an inductive ionization stage. Physics of Plasmas, 2020, 27 (2), pp.023518. 10.1063/1.5140241 . hal-03033131

\section{HAL Id: hal-03033131 \\ https://hal.science/hal-03033131}

Submitted on 1 Dec 2020

HAL is a multi-disciplinary open access archive for the deposit and dissemination of scientific research documents, whether they are published or not. The documents may come from teaching and research institutions in France or abroad, or from public or private research centers.
L'archive ouverte pluridisciplinaire HAL, est destinée au dépôt et à la diffusion de documents scientifiques de niveau recherche, publiés ou non, émanant des établissements d'enseignement et de recherche français ou étrangers, des laboratoires publics ou privés. 


\title{
Experimental characterization of ID-Hall, a double-stage Hall thruster with an inductive ionization stage.
}

\author{
A. Martín Ortega ${ }^{1}$ A. Guglielmi, ${ }^{1}$ F. Gaboriau, ${ }^{1}$ C. Boniface,${ }^{2}$ and J.P. Boeuf ${ }^{1}$ \\ 1) LAPLACE, Université de Toulouse, CNRS, INPT, UPS, 118 Route de Narbonne, 31062 Toulouse, \\ France \\ ${ }^{2)}$ Centre National d'Etudes Spatiales (CNES), 18 avenue Edouard Belin, 31401, Toulouse, \\ France
}

(Dated: 15 November 2019)

We present experimental results on the characterization of ID-Hall, a Double Stage Hall Thruster with an inductively coupled magnetized ionization stage. This first experimental prototype of ID-Hall operates in a low power regime, typically below $400 \mathrm{~W}$. The purpose of this work is to study the properties of ID-Hall and provide directions for optimization, but also and more generally, to study the relevance of the double-stage concept with respect to important physical issues such as current oscillations and anomalous electron transport. We present experimental measurements of the current voltage characteristics, extracted ion beam current (Faraday cup measurements), and ion energy and angular distribution functions (RPA measurements) in a single stage and double stage regime of operation, as a function of DC voltage and RF power. The results demonstrate that ions can be extracted from the inductive RF ionization stage even under conditions where the applied DC voltage is not sufficient to ionize the gas flow, i.e. that ID-Hall can indeed operate in a double stage regime. At intermediate voltages the overshoot observed in the current-voltage characteristics of single stage thrusters disappears in double stage operation, indicating a decrease of anomalous electron transport. Time-resolved and time-averaged ion energy distribution measurements show a coupling between ion energy, breathing mode oscillations and the magnetic configuration of ID-Hall.

\section{INTRODUCTION}

In Double Stage Hall Thrusters (DSHTs), a separate ionization source is placed upstream of the magnetic barrier in order to allow separate control of thrust (i.e. ionization) and specific impulse (i.e. ion acceleration). The concept of DSHT could also be useful for operation of the thruster with alternative propellants more difficult to ionize than xenon (krypton, argon, air breathing thrusters). A variety of double stage Hall thruster designs have been proposed ${ }^{1-8}$ but the relevance of the concept has not really been demonstrated ${ }^{9}$. The ion extraction area of a Hall thruster is much smaller than that of a gridded thruster so that efficient confinement of the plasma in the ionization stage and plasma generation as close as possible to the acceleration region are essential aspects of an efficient DSHT (the effective wall loss area in the ionization stage, taking into account magnetic confinement, should be small with respect to the channel extraction area). We have recently introduced a new design of DSHT, called ID-Hall ${ }^{9-11}$ (for Inductively coupled Double stage Hall thruster, patent pending) where the ionization stage is a magnetized RF inductively coupled plasma source with an RF coil inside the inner cylinder of the thruster (figure 1). The dimensions of the first experimental prototype of ID-Hall described here are such that the thruster can operate at a total power below 300-400 W. In this paper we present an experimental characterization of ID-Hall operating in single stage and double stage regimes. Measured thruster characteristic as a function of DC voltage in the acceleration stage and RF power in the ionization stage, including V-I curves, Faraday cup measurements of the extracted ion current and RPA measurements of the ion energy distribution function are discussed below. The ID-Hall design is briefly described in section II. The experimental set-up and diagnostics are presented in section III, and the main results

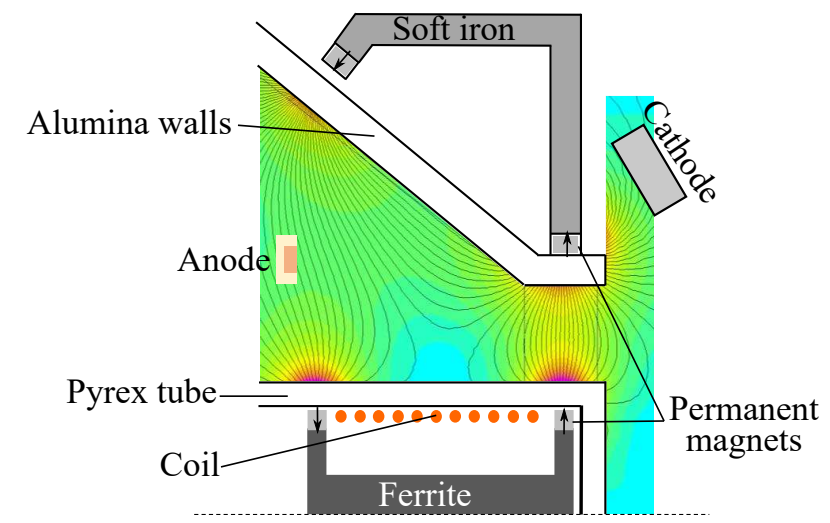

FIG. 1. Schematic of the ID-Hall thruster prototype used in this study, showing the magnetic field lines and intensity.

are discussed in section IV.

\section{ID-HALL}

The characteristic feature of ID-Hall is its ionization source, an inductively coupled plasma generated by an induction coil placed inside the central cylinder. This kind of source has been shown to provide a dense plasma confined in the form of a torus around the induction coil ${ }^{10}$. In the prototype used for this work, the coil is $25 \mathrm{~mm}$ long and $20 \mathrm{~mm}$ diameter, with 11 
turns of a one-millimeter copper wire around a ceramic holder. A low-loss ferrite cylinder of $100 \mathrm{~mm}$ diameter is placed at the center of the coil, to act as a magnetic conductor both to the DC magnetic field creating the magnetic barrier and the RF field of the inductive source. Two ferrite discs of $22 \mathrm{~mm}$ diameter are placed on both extremities of the induction coil, with $1 \mathrm{~mm}$ thick samarium-cobalt (SmCo) magnets placed around the ferrite discs. The choice of SmCo magnets was made due to the elevated maximum operation temperature of this material; SmCo magnets are commonly used in permanent magnet Hall thrusters $^{12}$ ? The assembly of inductive coil, ferrite and magnets is contained inside a $26 \mathrm{~mm}$ diameter Pyrex tube, which is also the inner wall of the thruster. The outer wall is made of alumina, and it can be divided in a cylindrical channel of $46 \mathrm{~mm}$ diameter and $10 \mathrm{~mm}$ length, and a conical ionization chamber of $30 \mathrm{~mm}$ length and an outer wall of increasing diameter up to $92 \mathrm{~mm}$ and the base of the cone. Two sets of SmCo magnets are placed immediately behind the channel wall and at the end of the conical wall. Both sets of magnets are kept in place by an L-shaped soft iron piece which also closes the DC magnetic circuit. The combination of the conical wall and the positioning of the magnets create an ionization chamber with 4 magnetic cusps and magnetic field lines parallel to the walls, reducing the ion losses at the walls and increasing the plasma density. In addition to the magnetic barrier of the thruster channel, a second magnetic barrier is created at the base of the ionization chamber. A schematic of the ID-Hall design and its magnetic field is shown in figure 1. The magnetic field configuration of this prototype is slightly different from those used in previous simulations ${ }^{9}$, due to the use of ferrites in the RF source instead of soft iron. The use of ferrites prevents the appearance of eddy currents in the magnetic circuit and therefore reduces the power losses. However, the ferrites have a lower saturation magnetic field than soft iron (around $500 \mathrm{mT}$ vs. $2 \mathrm{~T}$ ); as a consequence, the field lines are no longer parallel to the inner wall and instead the region of weak magnetic field is closer to the inner wall than when soft iron is used. The expected effect is a less efficient confinement and larger plasma losses to the inner wall. The zero magnetic field point should be moved radially away from the inner cylinder in future prototypes.

The anode consists of a copper disc of $40 \mathrm{~mm}$ diameter and $4 \mathrm{~mm}$ width, covered on the back side and on the inner and outer diameters by an insulating ceramic, so that only the front side of the disc is exposed to the plasma. It is placed at the maximum of the second magnetic barrier, so that the surface of the disc is approximately parallel to the magnetic field lines. Xenon is injected at the base of the ionization chamber after passing through a disc of porous ceramics. The cathode used in this study was provided by ICARE ${ }^{13}$. It is a hollow cathode with $\mathrm{L} \mathrm{LaB}_{6}$ disc placed behind the aperture and heated by a $200 \mathrm{~W}$ tungsten filament. A starter is used to trigger the discharge but is not required during continuous operation of the thruster.

The RF power source is an RFG 50-600 from Coaxial Power Systems operating at $4 \mathrm{MHz}$. The reduced frequency (with respect to the standard $13.56 \mathrm{MHz}$ ) was chosen to reduce the capacitive coupling and the losses in the central fer- rite; a side-effect is the increase of the RF current and the ohmic losses in the copper wire ${ }^{14}$. The coupling efficiency of the source is globally improved when operating at $4 \mathrm{MHz}$ instead of $13.56 \mathrm{MHz}$.

\section{EXPERIMENTAL SETUP}

\section{A. Vacuum chamber and experimental conditions}

In this study the ID-Hall prototype was fixed at one end of a vacuum vessel of $1 \mathrm{~m}$ length and $0.5 \mathrm{~m}$ diameter (former GREMI vacuum vessel ${ }^{12,15}$ ). The vessel is equipped with three turbo-molecular pumps providing a total pumping speed of $4200 \mathrm{l} / \mathrm{s}$ once the transparency of the protection grids is taken into account. Quartz windows placed at the opposite extremity of the chamber and at one side of the vessel provide frontal and lateral view of ID-Hall. All measurements presented here were performed with a Xe flow of $6 \mathrm{sccm}$ through the anode side and $1.5 \mathrm{sccm}$ through the cathode; under these conditions the background pressure was $6 \cdot 10^{-5}$ mbar. The ensemble of anode, cathode and starter/keeper together with their power sources floated with respect to the grounded chamber walls. The discharge current was obtained by measuring the voltage drop across a test resistance of $1.8 \Omega$ in the anode side, by means of a differential probe and a digital oscilloscope. The discharge current oscillations were also measured with a Rogowsky coil. The potential difference between cathode and ground was measured using a digital multimeter.

\section{B. Faraday probe measurements}

The Faraday probe used in this study consisted in two collector discs of $25 \mathrm{~mm}$ diameter on opposite sides of the probe, each one surrounded by a guard ring of $2 \mathrm{~mm}$ width. During the measurements the collectors and guard rings were biased to $-40 \mathrm{~V}$ to repel the background electrons. The Faraday probe is mounted on a rotating arm of $225 \mathrm{~mm}$ length and can reach angles from +80 degrees to -45 degrees, taking the thruster symmetry axis as the angular reference. The arm is connected to an in-vacuum stepper motor and rotating assembly which allows angular steps as small as 0.5 degrees. An automatic control and acquisition system designed and built at LAPLACE allowed automatic angular scans with variable angular steps, recording the value of the angle and the current collected from both front and rear collector surfaces at each step. The use of two Arduino cards, connected via an optical link, allowed to keep the measuring card floating so that it can measure a small potential drop (less than $3.3 \mathrm{~V}$ ) over a probe resistance on the high-voltage side of the circuit. A diagram of the control and acquisition system is shown in figure 2 .

The use of a double Faraday probe allowed us to measure the ion current due to the background plasma with the back collector and subtract it from the total ion current measured with the front collector. However, while the current in the front collector is independent of the probe voltage once 


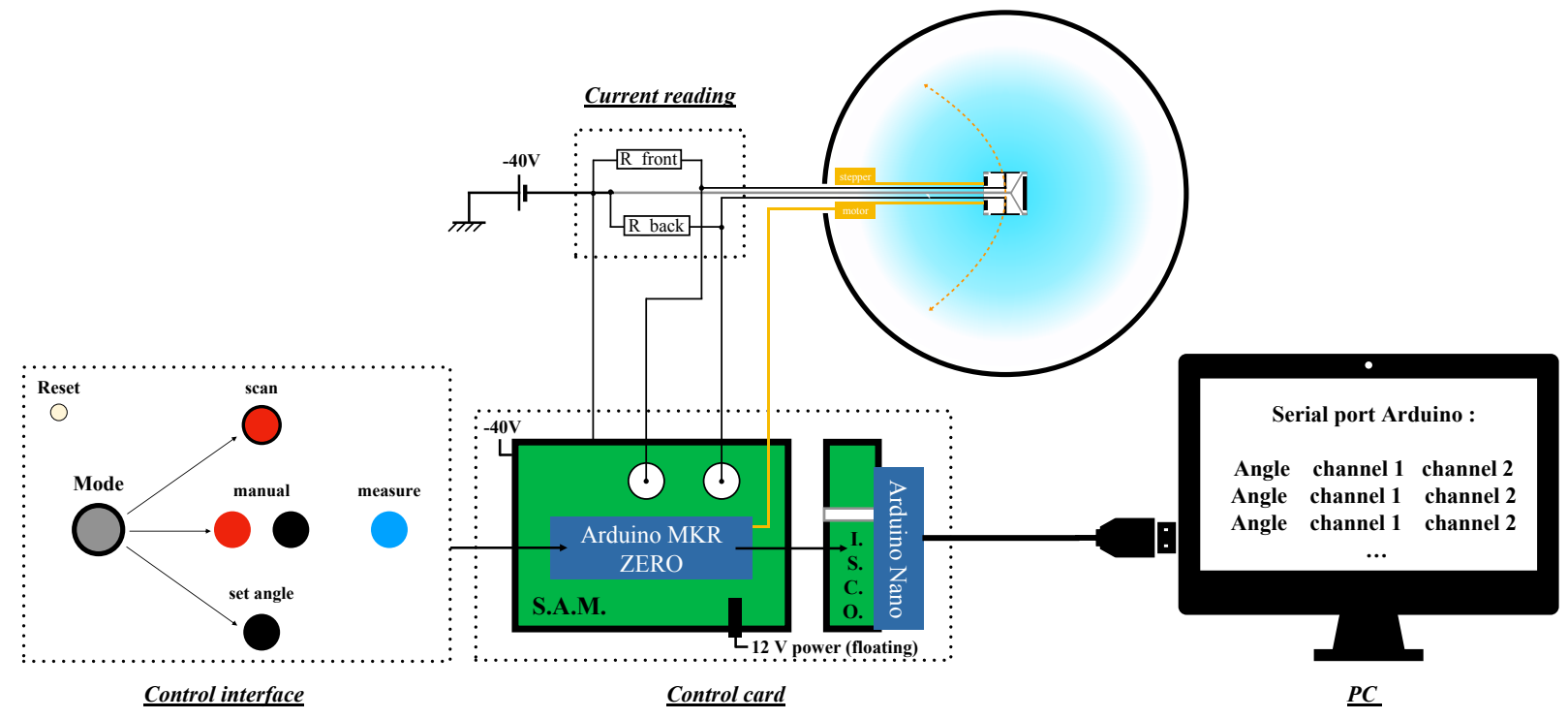

FIG. 2. Schematic of the Faraday probe automatic acquisition system. The potential drop across the sensing resistances (R_front and R_back) is read by a floating Arduino card (S.A.M.); the information is passed via an optical link to a second card (I.S.C.O.) connected to a computer by a serial port.
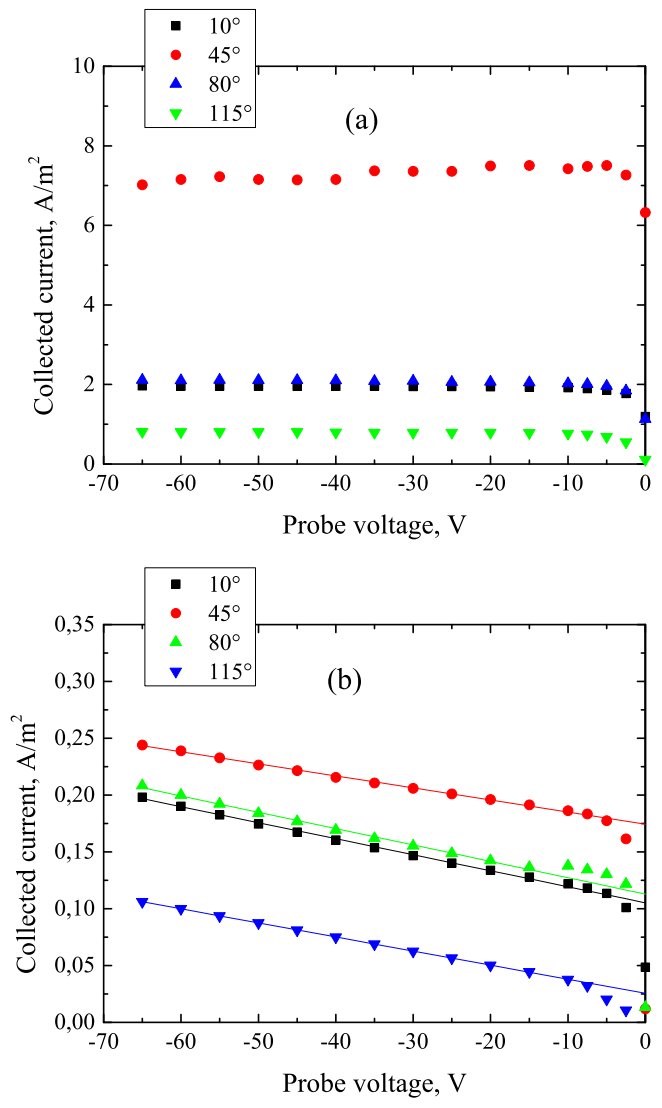

FIG. 3. (a) Current at the front collector of the Faraday probe as a function of probe voltage for different angles. (b) Current at the back collector of the Faraday probe as a function of probe voltage for different angles (symbols). Lines: linear fit of the points for $\mathrm{V} \leq$ $10 \mathrm{~V}$. the background electrons are repelled $(\mathrm{V}<-5 \mathrm{~V}$, figure $3 \mathrm{a})$, the sheath expansion on the back side leads to a continuous increase of the collected current as a function of the bias potential $^{16}$ as can be seen in $3 \mathrm{~b}$. To account for the sheath expansion, a number of I-V curves were taken at several angular positions; the ion current corresponding to the collector surface was taken as the extrapolation to $0 \mathrm{~V}$ of a linear fitting of the measured I-V curves. The fitting coefficients obtained were used later to obtain the background current from measurements at the fixed probe bias of $-40 \mathrm{~V}$.

Once the angular distribution of the ion current is known, the total ion current can be calculated as

$$
I_{i}=2 \pi R \int_{0}^{\pi / 2} J_{i}(\theta) \sin (\theta) d \theta
$$

where $J_{i}$ is the ion current density, $\Theta$ the azimuthal angle and $R$ the distance from the probe to the thruster, coincident with the probe rotation axis. In practice, the integral is not calculated up to $\pi / 2$ but only to the largest available angle, i.e. +80 degrees. However, we expect this to be a good approximation to the true ion current, and in any case it will allow to examine the variations of ion current as a function of the different operating conditions. Also, given the relatively high background pressure, we expect the ion current at large angles to be highly overestimated due to charge exchange collisions $(\mathrm{CEX})^{17}$, so that the measurements at larger angles would not be representative of the true plume ion current in vacuum.

\section{Time-averaged RPA measurements}

To measure the IEDF of the plume ions, we used a standard 4-grid RPA developed in the group of A. Bouchoule at the GREMI laboratory of the University of Orleans in 1998; 
its design and operating conditions are reported in detail in the $\mathrm{PhD}$ thesis of C. Philippe-Kaldec. The RPA was placed on a rotating arm $55 \mathrm{~cm}$ away from the thruster ${ }^{15}$. However, we measured the IEDF only at the symmetry axis since the angular distribution of ions was already measured by the Faraday probe. The rejecting grid voltages were set to $-35 \mathrm{~V}$ and $-40 \mathrm{~V}$ to prevent background electrons from entering the RPA and to prevent secondary electrons from being emitted away from the collector surface. The voltage on the analyzing grid was set proportional to the voltage of a function generator providing a triangular signal. The upper and lower limits of the sweeping voltage were adjusted at each operating condition to obtain the best energy resolution possible. The signal frequency was set to $500 \mathrm{~Hz}$, much lower than any oscillation found in the discharge current signal. The signals from the function generator and the collector of the RPA were recorded using a digital oscilloscope. A band-reject filter centered at $4 \mathrm{MHz}$ was added to the collector circuit to suppress the noise from the ionization source when operating in double stage. To further reduce the noise (both single and double stage measurements), each measurement was averaged over 128 tension sweeps, with the signal from the function generator used to trigger and synchronize the acquisitions. The derivative of the recorded signal with respect to the analyzing voltage was obtained using a Savitzky-Golay filter to obtain the IEDF.

\section{Time-dependent RPA measurements}

In addition to the time-averaged RPA measurements, we performed time-dependent RPA measurements to study the evolution of the IEDF in the presence of breathing mode oscillations in the discharge current. These oscillations have a frequency between 10 and $30 \mathrm{kHz}$, and were regular and repetitive enough to be used as a reference to trigger and synchronize the acquisitions made with the digital oscilloscope. For each acquisition, the analyzing voltage was fixed and the collector signal was collected over one or more oscillations. The process was repeated for discrete steps of the analyzing voltage, usually a $5 \mathrm{~V}$ step but occasionally a smaller step was used whenever the signal changed rapidly. Because the trigger condition was identical for all the acquisitions, we can align the acquisitions to obtain a matrix of $n x m$ size, where $n$ is the number of voltage steps and $m$ the size length of each acquisition. Then, the IEDF at each time $t$ can be obtained using a Savitzky-Golay filter along the first dimension $(n)$, for each different $t$ time $(m)$. This method gives the IEDF measured at the RPA location. We can also calculate the IEDF at the exhaust plane of the thruster by calculating the time of flight of the ions over the distance $D_{R P A}$ from the thruster to the RPA $(55 \mathrm{~cm})$. This time depends on the ion speed $v_{E_{c}}=\sqrt{2 E_{c} / M_{i}}$ where $E_{c}$ is the measured ion kinetic energy and $M_{i}$ the ion mass. For each energy of the measured IEDF, the time $t^{\prime}$ at which the ions left the thruster is deduced as $t^{\prime}=t-D_{R P A} / v_{E_{C}}$.

\section{E. Inductively coupled plasma source efficiency}

The efficiency of the inductive RF source was measured using a current-voltage probe connected to a digital oscilloscope. First, the RMS current and voltage were measured without plasma; in this configuration the power loss is due to ohmic dissipation in the copper wire and to magnetic losses in the ferrite core. Assuming that the power losses in the ferrite are negligible, all the losses are due to ohmic heating of the copper wire, whose resistance can be calculated as $R_{\text {wire }}=$ $P / I_{R M S}^{2}$, where $P$ is the injected power measured by the RF source and $I_{R M S}$ is the root mean square of the RF current. The resistance was found to be $R_{\text {wire }}=2.5 \Omega$. Then, the power coupled to the plasma will be the difference between the injected power and the heating losses: $P_{\text {plasma }}=P_{\text {tot }}-R_{\text {wire }} I_{R M S}^{2}$

\section{RESULTS AND DISCUSSION}

\section{A. Single stage characterization}

ID-Hall was first characterized in single-stage operation, i.e. without power in the inductive plasma source. Figure 4 shows the current voltage characteristics of ID-HALL operating in a single stage mode at $6 \mathrm{sccm}$. To verify the reproducibility of the results, several voltage sweeps were performed at different times: right after the starting of the discharge ("cold"), after a few minutes of operation ("warm") and finally at the end of the measurements ("hot"). In addition to these sweeps, the angular ion current distribution and the IEDF were measured every $10 \mathrm{~V}$, together with the discharge current waveform. There were some variations of the discharge current between the different sweeps, but the general features were well reproduced in all three cases. One can distinguish two distinct discharge regions: a first region below $190 \mathrm{~V}$, where the discharge current passes through a maximum around $0.68 \mathrm{~A}$ and decreases slowly to $0.58 \mathrm{~A}$ with increasing voltage, and a second region above $190 \mathrm{~V}$ where the current increases continuously with increasing voltage. This continuous increase at higher voltages is thought to be caused by secondary electron emission from the alumina walls and is consistent with previous observations ${ }^{18}$ of Hall thruster I-V characteristics with alumina walls. This is in contrast with the I-V characteristics of Hall thrusters with $\mathrm{BN}$ or $\mathrm{BN}-\mathrm{SiO}_{2}$ walls which exhibit a long region of constant current above $150 \mathrm{~V}^{18}$. The current oscillations below and above $190 \mathrm{~V}$ are also very different, as can be seen in figure 5. Breathing mode oscillations are observed on the discharge current below $190 \mathrm{~V}$, with a well-defined frequency increasing from $8 \mathrm{kHz}$ at $100 \mathrm{~V}$ up to $15 \mathrm{kHz}$ at $180 \mathrm{~V}$. The amplitude of the oscillations (figure 5), is between 2 and 3 times the mean current in this part of the I-V characteristics. At higher voltages the breathing mode oscillations disappear, and the random fluctuations of the current have much smaller amplitudes. We call this regime "continuous mode".

The results obtained with the Faraday probe show that the ion current (empty squares in figure 4) increases with voltage up to a maximum of $0.39 \mathrm{~A}$ at $170 \mathrm{~V}$ (the maximum $\mathrm{Xe}^{+}$cur- 


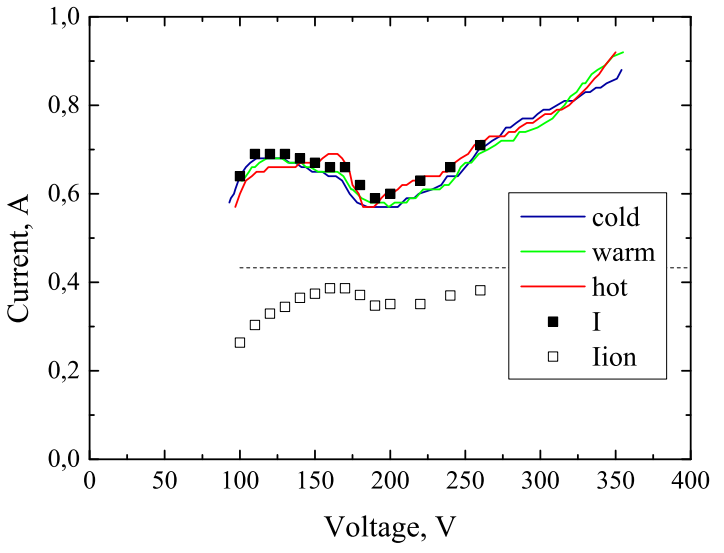

FIG. 4. Lines: I-V sweeps performed just after initiation of the discharge ("cold"), after 5 minutes of operation ("warm") and after two hours ("hot"). Single stage operation at $6 \mathrm{sccm}$. Open square symbols: discharge current measured simultaneously with the Faraday probe scans. The dashed line corresponds to the maximum $\mathrm{Xe}^{+}$ion current at $6 \mathrm{sccm}$.

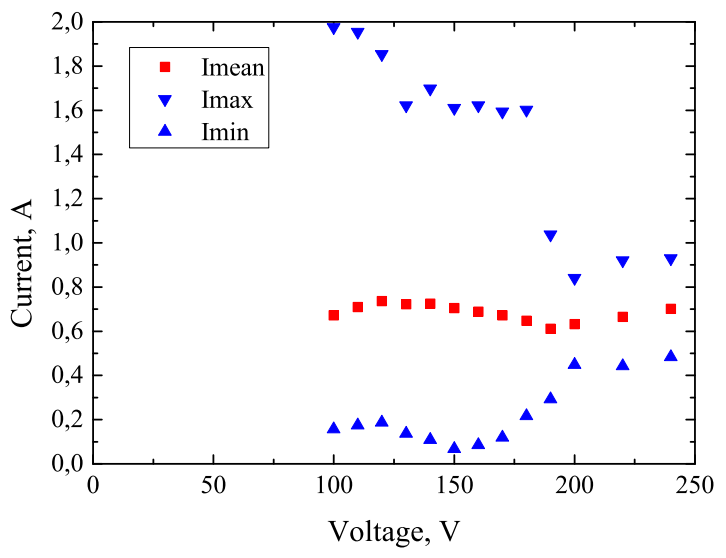

FIG. 5. Mean discharge current (square symbols) and maxima (down triangle symbols) and minima (up triangle symbols) of the oscillations as a function of the discharge voltage in single stage operation at 6 sccm,. Large amplitude "breathing mode oscillations" are present below $190 \mathrm{~V}$ while a quasi "continuous mode" exists above $190 \mathrm{~V}$.

rent at $6 \mathrm{sccm}$ is $0.43 \mathrm{~A}$ ). Above this voltage, the ion current drops together with the total current, then slightly increases as the voltage increases further. These differences come from differences on the ion current at large angles; the angular distributions (figure 6) have a similar maximum ion current and vary only slightly at large angles. The divergence angle, taken as the half-angle which contains $90 \%$ of the ion current, is also similar: for the two distributions of figure 6 , it is 65 degrees at $170 \mathrm{~V}$ and 63 degrees at $220 \mathrm{~V}$. Note that the drop in the total ion current coincides with the change of oscillation mode observed in the discharge current.

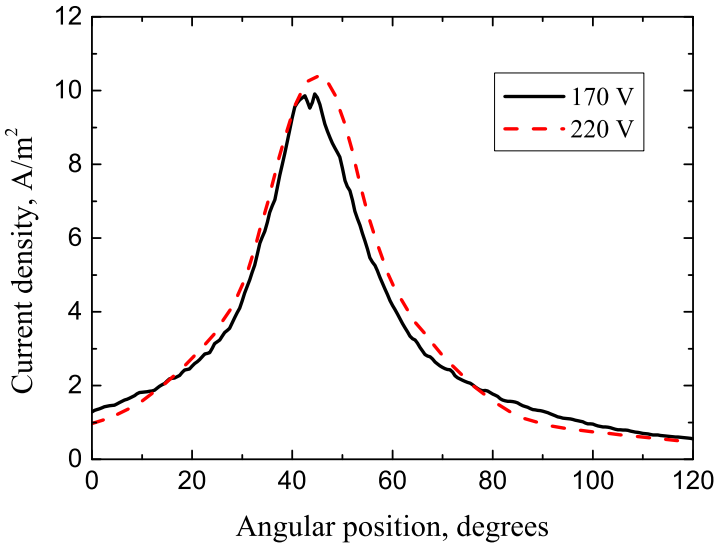

FIG. 6. Angular distribution of the ion current in the plume in single stage operation at $6 \mathrm{sccm}$, with $(170 \mathrm{~V})$ or without $(220 \mathrm{~V})$ the presence of breathing mode oscillations in the discharge current.

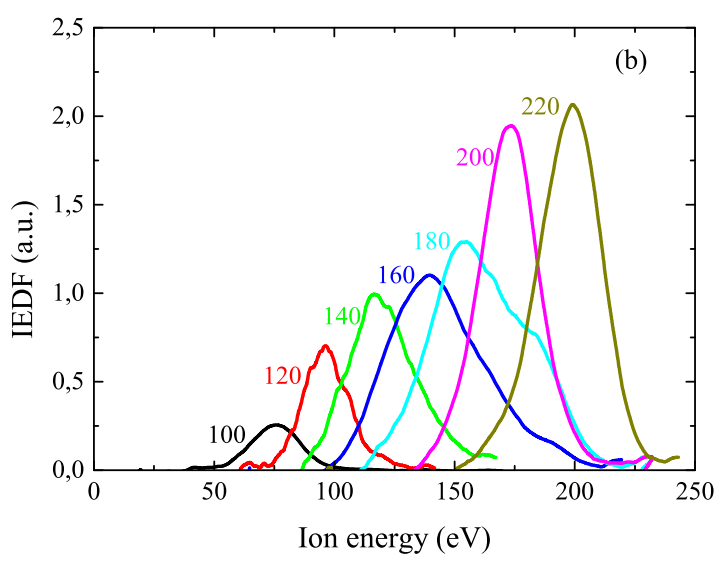

FIG. 7. IEDF measured by RPA at the symmetry axis of the thruster for different discharge voltages, in single stage operation. The label next to each line indicates the discharge voltage.

The RPA measurements show two distinct IEDF profiles depending on whether breathing oscillations are present or not, as shown in figure 7. In the oscillatory mode, the IEDF has a larger width, with the peak being slightly lower than the discharge voltage. Above $180 \mathrm{~V}$, in the continuous mode, the distributions become much narrower, again with the maxima being lower than the corresponding discharge voltage. The energy shift between the peak of the distribution and the discharge voltage is not due to the cathode potential, which was measured simultaneously and found to be only of around -5 $\mathrm{V}$, while the difference between the maxima and the discharge voltage is about $20 \mathrm{~V}$. This difference may be due to the presence of the second magnetic barrier in the anode region at the end of the ionization stage of ID-Hall. Under some conditions, a potential drop may form in this region because of the smaller electron mobility, leading to a plasma potential lower than the 
anode potential and to the lowering the maximum ion energy at exhaust. The presence of such potential drop in the anode region in single stage operation has been already predicted in simulations of ID-Hall using the hybrid model HALLIS ${ }^{9,19}$.

The time evolution of the IEDF in the presence of breathing mode oscillations is shown in figure $8 \mathrm{a}$, for a discharge voltage of $150 \mathrm{~V}$. It can be seen that the ion energy is modulated in time, with an energy around $120 \mathrm{eV}$ before the maximum ion current, increasing energy together with the peak ion current (absolute maximum of the IEDF at $8 \mu \mathrm{s}$ ) up to a maximum energy slightly above $150 \mathrm{eV}$ when the ion current decreases again before restarting the cycle. The shift in the maximum of the instantaneous distribution can be seen more clearly in figure $8 \mathrm{~b}$, where the instantaneous distributions corresponding to specific times are shown. Here, we can see the shift of the maximum of the distribution from lower to higher energies as time increases; note that the last shown distribution $(\mathrm{t}=22$ $\mu \mathrm{s})$ has a peak energy higher than the discharge voltage. This energy shift in time explains the broad time-averaged distributions shown previously in figure 7 for the cases where breathing mode oscillations were present. In the absence of breathing mode oscillations, there is no modulation of the IEDF and the width of the distribution becomes narrower.

To help explaining the origin of this modulation of the IEDF in the presence of the breathing mode, we used a theoretical model of ID-Hall using the simulation software HALLIS. This software has already been used to estimate the performance and the relevance of the concept of ID-Hall ${ }^{9}$. Here we make only a brief introduction to HALLIS; a more detailed description of HALLIS can be found in Ref. 20. HALLIS is a twodimensional (2D) hybrid particle-fluid model in the R-Z plane (therefore assumes azimuthal symmetry) where electrons are treated as a fluid while ions and atoms are described by quasiparticles. To account for the anomalous electron transport across the magnetic barrier, the model uses effective collision frequencies corresponding to the roles of turbulent transport and electron-wall interaction. These effective collision frequencies are controlled by adjustable coefficients that can be varied between simulations.

To obtain breathing mode oscillations in the simulations of ID-Hall, we have to set rather specific values of the anomalous transport coefficients, discharge voltage and gas flow; a deviation from those parameters would result in a continuous mode with no discharge current oscillations. The results shown here were obtained with a mass flow of $1 \mathrm{mg} / \mathrm{s}$ and a discharge voltage of $250 \mathrm{~V}$. Under these conditions, the oscillation frequency was of $8 \mathrm{kHz}$, lower than the $15-20 \mathrm{kHz}$ observed experimentally. Nevertheless, the results of the simulation, shown in figure 9, allow for explaining the modulation of the IEDF. Figure 1a shows a typical current oscillation, with an initial peak of current followed by a longer plateau and a final drop to zero before restarting the cycle. Figure 9 shows the magnetic field magnitude along the center of the channel; we see the first barrier between $\mathrm{z}=30$ and $35 \mathrm{~mm}$, near the exit plane of ID-Hall, and the second one in front of the anode at $\mathrm{z}=5 \mathrm{~mm}$. Figures $9 \mathrm{c}$ and $9 \mathrm{~d}$ show respectively the plasma potential and the electron density along the center of the channel, for different times of the oscillation. At zero current (label
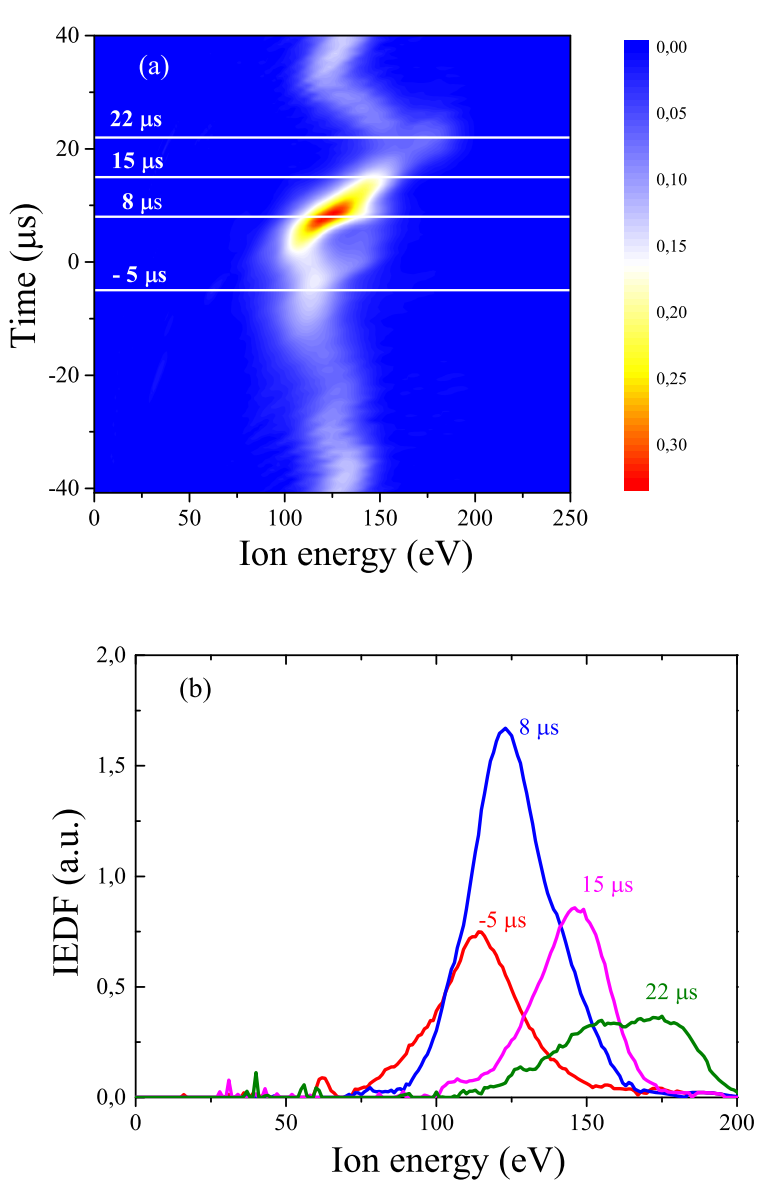

FIG. 8. (a) Time evolution of IEDF at the exhaust plane for a $150 \mathrm{~V}$ discharge voltage in single stage, without power in the ionization source. (b) Ion energy distribution at 4 different times corresponding to the white lines of figure 8a. The xenon flow rate is $6 \mathrm{sccm}$.

1), the electron density is near zero and the plasma potential has two drops, located at the two magnetic barriers due to the lower plasma conductivity in those regions. At this point the ionization starts in the outer barrier, the current starts increasing and the electron density in the chamber increases (labels 2-3), filling the ionization chamber with plasma. The increase in electron density also increases the electron conductivity on the second magnetic barrier, near the anode, so that the potential drop decreases accordingly. The ions accelerated at this time have an energy smaller than the discharge voltage, the difference being the potential drop near the anode. After the current peak, during the plateau of the current oscillation (labels 4 to 7) the electron density near the anode is high enough to make disappear the potential drop in the second magnetic barrier, so that the ions are accelerated by the full discharge voltage in the first magnetic barrier. During the plateau the electron density decreases slowly until dropping to zero at the end of the oscillation, when the potential drop in the second magnetic barrier reappears and the cycle restarts. In this simulation, the behavior of the potential drop in the first magnetic 

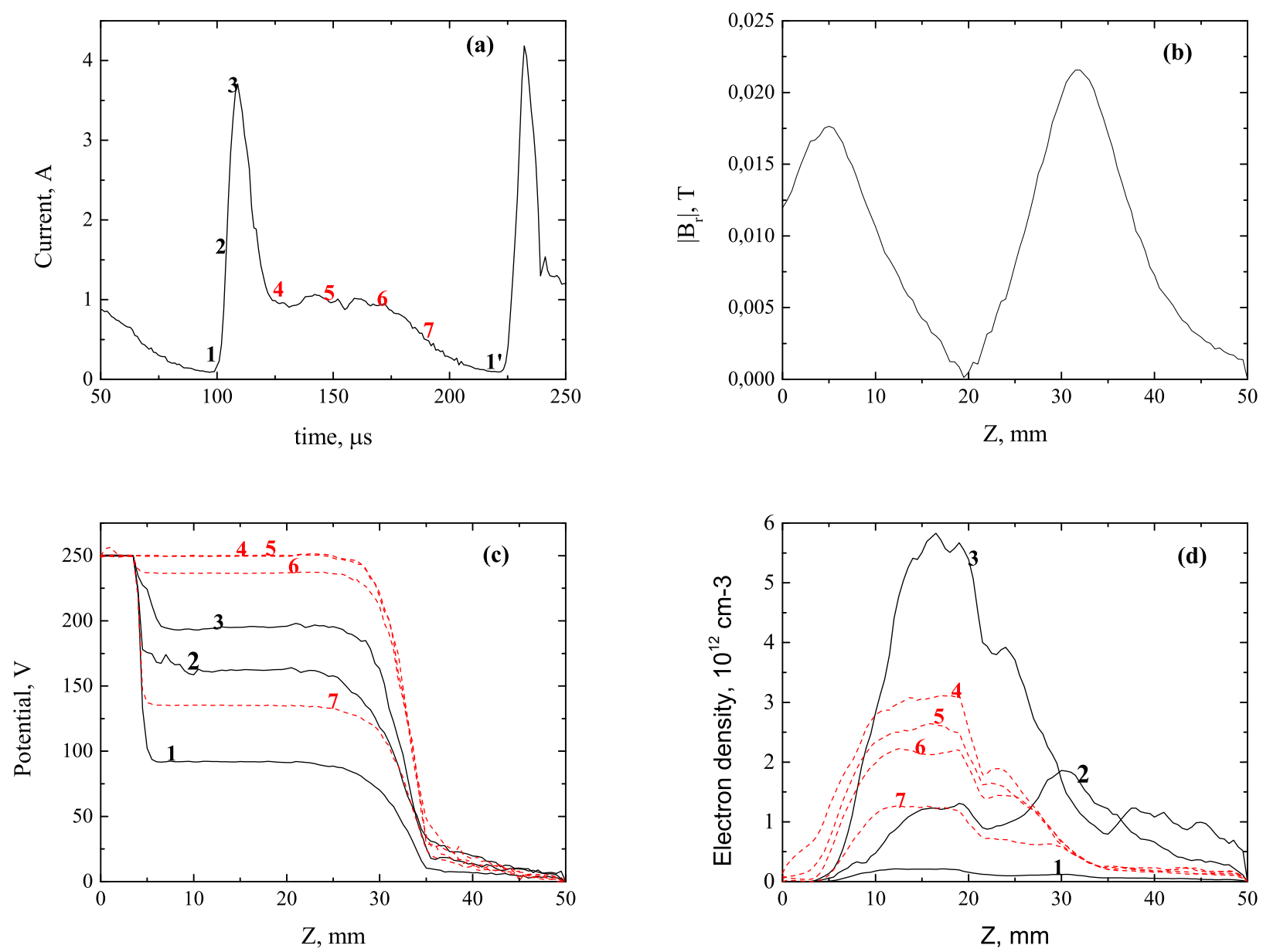

FIG. 9. Simulation results of ID-Hall assuming a mass flow of $1 \mathrm{mg} / \mathrm{s}$ and a discharge voltage of $250 \mathrm{~V}$. (a) Discharge current oscillation. (b) Absolute value of the radial magnetic field along the center of the channel. (c) Plasma potential along the center of the channel. (d) Electron density along the center of the channel. The indexes in figure (a) indicate the time values of the curves plotted in (c) and (d). The anode position is at $\mathrm{Z}=3 \mathrm{~mm}$ and the exhaust plane at $\mathrm{Z}=35 \mathrm{~mm}$.

barrier (starting lower than the applied voltage and later increasing to its maximum value) matches the one observed experimentally by the time-dependent IEDF measurements. We can conclude therefore that the energy shift in the ion energy is due to the coupling between the breathing mode oscillation and the potential drop in the first magnetic barrier, due to the specific magnetic configuration of ID-Hall.

\section{B. Double stage characterization}

The characterization of ID-Hall in double stage was performed by making both sweeps of the injected RF power at fixed voltage, and sweeps of the discharge voltage for fixed RF power. During the power sweeps, the DC voltage was fixed from 0 to $200 \mathrm{~V}$ with steps of $50 \mathrm{~V}$. These sweeps started at $0 \mathrm{~W}$ when the voltage was $100 \mathrm{~V}$ and above, i.e. when single stage operation was possible. For lower voltages the sweeps started at the minimum RF power capable of sustain- ing the plasma. During the voltage sweeps, the RF power was fixed at $50 \mathrm{~W}, 100 \mathrm{~W}$ and $150 \mathrm{~W}$ with voltage steps of $10 \mathrm{~V}$. The minimum operating voltage at $50 \mathrm{~W}$ was $60 \mathrm{~V}$. At 100 $\mathrm{W}$ and $150 \mathrm{~W}$, it was possible to sustain the plasma with the RF plasma source alone, so that the voltage sweeps start at 0 V. As in the characterization at single stage, the xenon flow was $6 \mathrm{sccm}$ through the anode side and $1.5 \mathrm{sccm}$ through the cathode.

The fraction of the RF power coupled to the plasma is shown in figure 10, as a function of the total RF power given by the RF power supply. The coupling efficiency is higher than $70 \%$ when the plasma density is high enough; in particular, it reaches $75 \%$ at $50 \mathrm{~W}$ of RF power for voltages higher than $100 \mathrm{~V}$. For similar ICP plasma sources with the coil surrounded by the plasma, the power coupling efficiency can be as high as $90 \%{ }^{21}$ and optimization of the coupling efficiency for ID-Hall will be performed in future work.

The discharge and ion current of ID-Hall in double stage operation is shown in figure 11, as a function of the coupled 
RF power for different values of the discharge voltage. At 0 and $50 \mathrm{~V}$ there is a continuous increase in both discharge and ion current with increasing RF voltage. Note that below 50 $\mathrm{V}$ the voltage is too low for ionization in the magnetic barrier by electrons coming from the cathode (single stage operation is not possible, so the ions must have been generated in the ionization stage and accelerated in the magnetic barrier. This result shows that in this low voltage range, ID-Hall effectively works as a double-stage thruster, i.e. ions generated in the ionization stage are extracted and accelerated through the magnetic barrier. The lack of saturation in the ion current at $50 \mathrm{~V}$ indicates that more RF power (higher than $140 \mathrm{~W}$, the maximum applied in this work) or a better confinement in the ionization stage is required to achieve the maximum ion current $(0.43 \mathrm{~A})$. At higher discharge voltages $(100 \mathrm{~V}, 150 \mathrm{~V}$ and $200 \mathrm{~V}$ ) the main effect of the increase of RF power is the decrease of total discharge current; the ion current also decreases slightly but in a much less pronounced way. The result is a reduced electron current crossing the magnetic barrier. For instance, at $150 \mathrm{~V}$, the total current decreases from $0.68 \mathrm{~A}$ in single stage to $0.45 \mathrm{~A}$ at $125 \mathrm{~W}$ (coupled power), a decrease of $0.23 \mathrm{~A}$, while the ion current decreases only from $0.38 \mathrm{~A}$ to $0.30 \mathrm{~A}$; therefore, the electron current entering the channel must have decreased from $0.30 \mathrm{~A}$ in single stage to $0.15 \mathrm{~A}$ in double stage. This effect can be seen more clearly in figure 12 , where the $\mathrm{I}-\mathrm{V}$ characteristics and the ion current are shown for single stage and fixed RF power values in double stage. For voltages between 100 and $200 \mathrm{~V}$, the total current decreases as the RF power increases, much more so than the ion current. At voltages above $200 \mathrm{~V}$ there is no significant differences between single and double stage, and therefore no net gain from the addition of the ionization source. Note that above $200 \mathrm{~V}$ the wall conductivity increases due to the material (alumina), so the behavior may be different for different wall materials. Below $100 \mathrm{~V}$ operation in single stage is not possible, only in double stage for high enough values of RF power. If we assume that below $100 \mathrm{~V}$ there is no additional ionization in the magnetic barrier, we can estimate the energy cost per ion in the ionization stage. At $150 \mathrm{~W}$ of RF power and $50 \mathrm{~V}$, the ion current is $0.2 \mathrm{~A}$, which makes $150 \mathrm{~W} / 2 \mathrm{~A}=$ $750 \mathrm{eV} / \mathrm{ion}$. This cost is higher than typically found in gridded ion engines, indicating than the magnetic confinement in the ionization source can still be improved to limit ion losses.

The angular distributions of the ion current is shown in figure 13, for two different values of discharge voltage: $100 \mathrm{~V}$ in figure $13 \mathrm{a}$ and $200 \mathrm{~V}$ in figure $13 \mathrm{~b}$. At $100 \mathrm{~V}$, the increase of RF power results in an increase of the ion current at the center but not at large angles, therefore reducing the divergence of the plume. This can be attributed to he fact than the additional ions are created deep in the ionization chamber and as a consequence follow straighter trajectories. Note that the current at large angles remains almost unchanged for all RF powers. The contribution of the ion current at large angle to the total ion current is dominant so that the ion current does not change significantly with RF power (Figure 10) in spite of the lower divergence at higher RF power. For a discharge voltage of $200 \mathrm{~V}$ the trend is the opposite: the ion current at the center decreases with increasing RF power and therefore

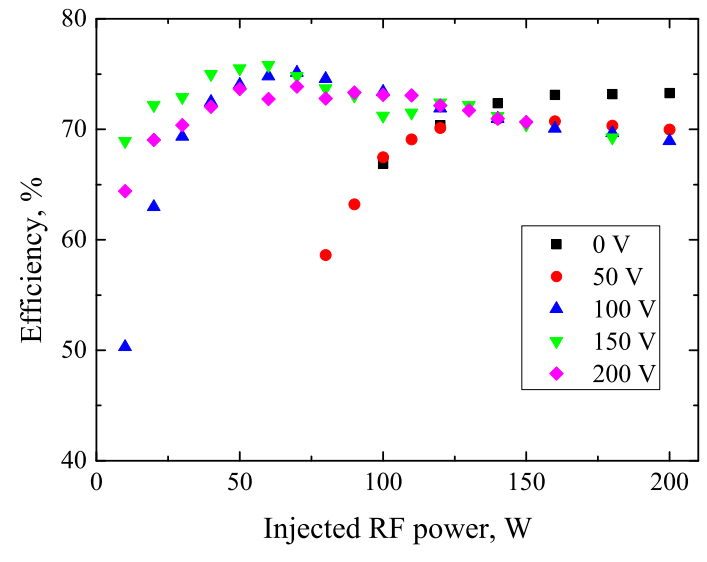

FIG. 10. RF source efficiency as a function of the injected power, for different values of the discharge voltage.

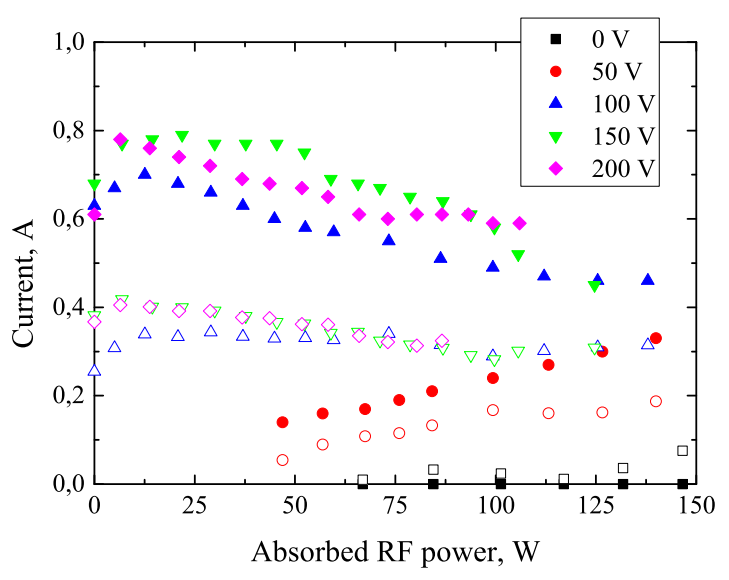

FIG. 11. Discharge current (full symbols) and ion current (open symbols) as a function of absorbed RF power, for different discharge voltages.

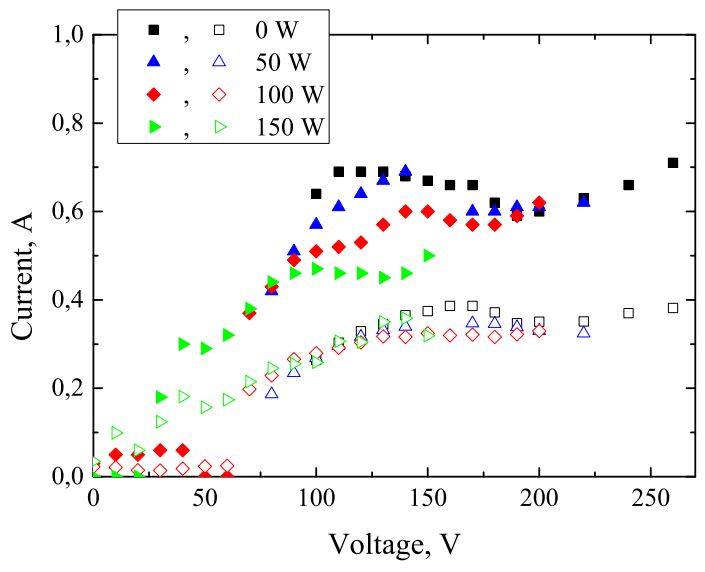

FIG. 12. I-V characteristics for different fixed RF injected power. Full symbols: discharge current. Open symbols: ion current. 

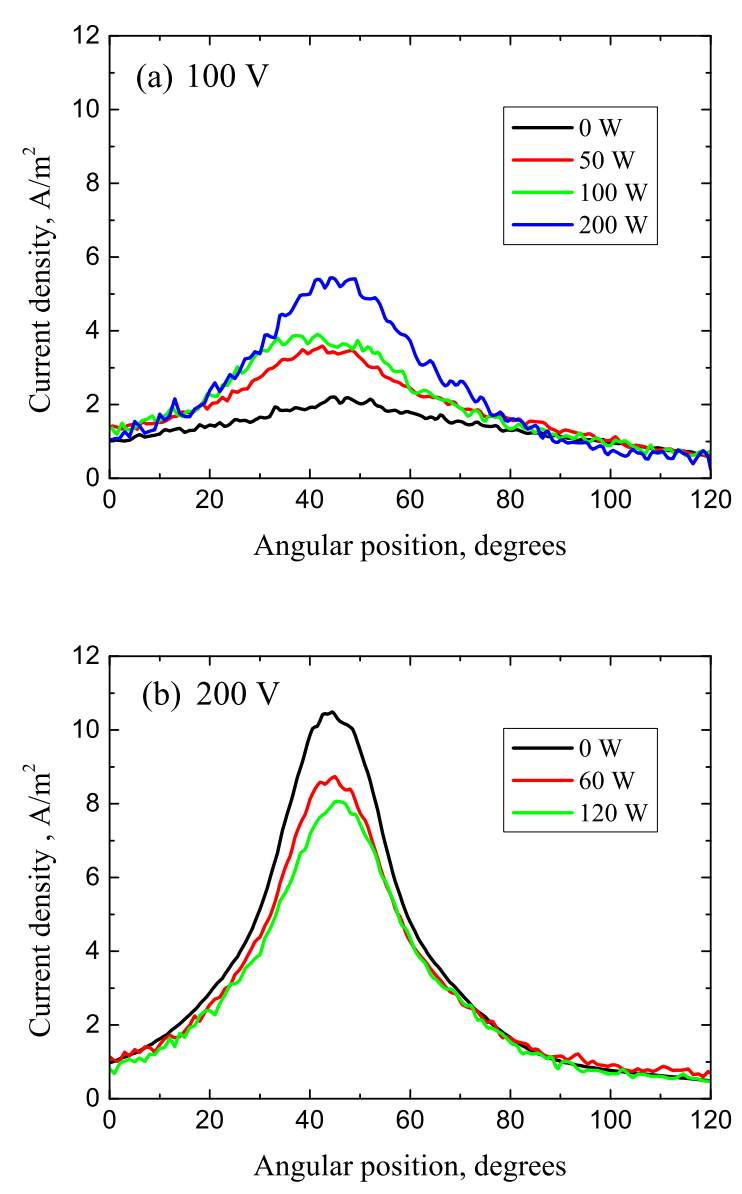

FIG. 13. Angular distributions of the ion current as a function of the $\mathrm{RF}$ injected power, (a), for a discharge voltage of $100 \mathrm{~V}$, and (b), for a discharge voltage of $200 \mathrm{~V}$.

the divergence of the plume increases, again without significant changes to the total ion current. This effect is not yet clearly understood.

We now look at the IEDF in double stage operation. Figure 14 shows the influence of the RF power on the IEDF for two different values of the discharge voltage. Interestingly, for a fixed discharge voltage, the energy of the ion beam is dependent on the RF power, and this clearly appears at low discharge voltages.For a discharge voltage of $100 \mathrm{~V}$ (figure 14a) the energy at the maximum of the IEDF increases with increasing RF power. The energy at the IEDF maximum is 75 $\mathrm{eV}$ at $0 \mathrm{~W}$ (single-stage) and increases gradually up to $120 \mathrm{eV}$ at $200 \mathrm{~W}$ of injected RF power (138 W absorbed power). This energy is actually higher than the discharge voltage, which means that the ions are generated in and accelerated from a region at a potential higher than the anode potential. Therefore, the plasma potential in the ionization chamber is above the anode potential and increases with increasing RF power. A possible explanation of this feature is that the electron mean energy in the chamber increases with the RF power. The electron temperature in a low-pressure plasma source at constant gas density depends mainly on the surface to volume ratio of the chamber (this results from the balance between ionization and losses), and not on the absorbed power. But in our conditions we expect the gas to be strongly depleted by ionization, so that the gas density decreases with increasing RF power, and therefore the ionization rate (and mean electron energy) should also increase. The plasma potential with respect to the walls in a low temperature non magnetized plasma is $\frac{1}{2} T_{e}\left(1+\ln \left(\frac{M}{n \pi m_{e}}\right)\right)$, where $M$ is the ion mass; for xenon this expression is approximately $5.8 T_{e}$, with $T_{e}$ in $\mathrm{eV}$. For an electron temperature of 5-6 eV, the plasma potential can me more than $30 \mathrm{eV}$, which is consistent with the result of figure 14a. In this case the reference wall is the anode; the apparent disappearance of the second magnetic barrier indicates that the electron density in this region is high enough to increase the electron conductivity, so that all the potential drop appears in the first magnetic barrier. The dissapearance of the potential drop in the second magnetic barrier in double stage operation was also predicted in previous simulations of ID-Hall ${ }^{9}$.

At higher voltages, i.e. $200 \mathrm{~V}$ (figure 14b), the mean energy does not increase continuously but shifts from $181 \mathrm{eV}$ in single stage to $203 \mathrm{eV}$ in double stage, with the width of the distribution first increasing and then decreasing as the RF power increases. This apparently complex behavior is related to the presence or absence of breathing mode oscillations, similar to those observed in single stage. The amplitude of the oscillations is shown in figure 15 for $100 \mathrm{~V}$ (figure 15a) and $200 \mathrm{~V}$ (15b). At $100 \mathrm{~V}$ the breathing mode oscillations are present in single stage and are suppressed as the RF power in the ionization stage increases; for an RF power above $40 \mathrm{~W}$ there is no breathing mode. At $200 \mathrm{~V}$ the behavior is more complex, with a continuous mode in single stage which corresponds to the narrow distribution seen in figure $14 \mathrm{~b}$; the breathing mode oscillations appear with an RF power as small as $10 \mathrm{~W}$ and are then reduces its amplitude for increasing RF power. As in single stage operation, the larger width of the IEDF is due to the presence of oscillations of the plasma potential with respect to the anode potential when breathing oscillations are present.

The operation domains of breathing oscillations ("breathing mode") and small fluctuations ("continuous mode"), together with the regions where operation is not possible, are represented in figure 16. Here we can see that at high enough RF power, operation is possible at any voltage, and the breathing mode oscillations are suppressed. Note that the small region above $200 \mathrm{~V}$ in single stage is also a "continuous mode".

\section{Estimated thrusts and efficiency}

The combined measurements of the IEDF and the angular distribution of ions in the plume allow us to calculate the thrust of ID-Hall under the different operation conditions. Assuming that the IEDF is independent of the angular direction, the total thrust can be decomposed as contributions from the mean ion speed $\langle v\rangle$, mean cosinus $\langle\cos (\theta)\rangle$ ans total ion flux $\dot{m}$ :

$$
T=\dot{m}\langle v\rangle\langle\cos (\theta)\rangle
$$



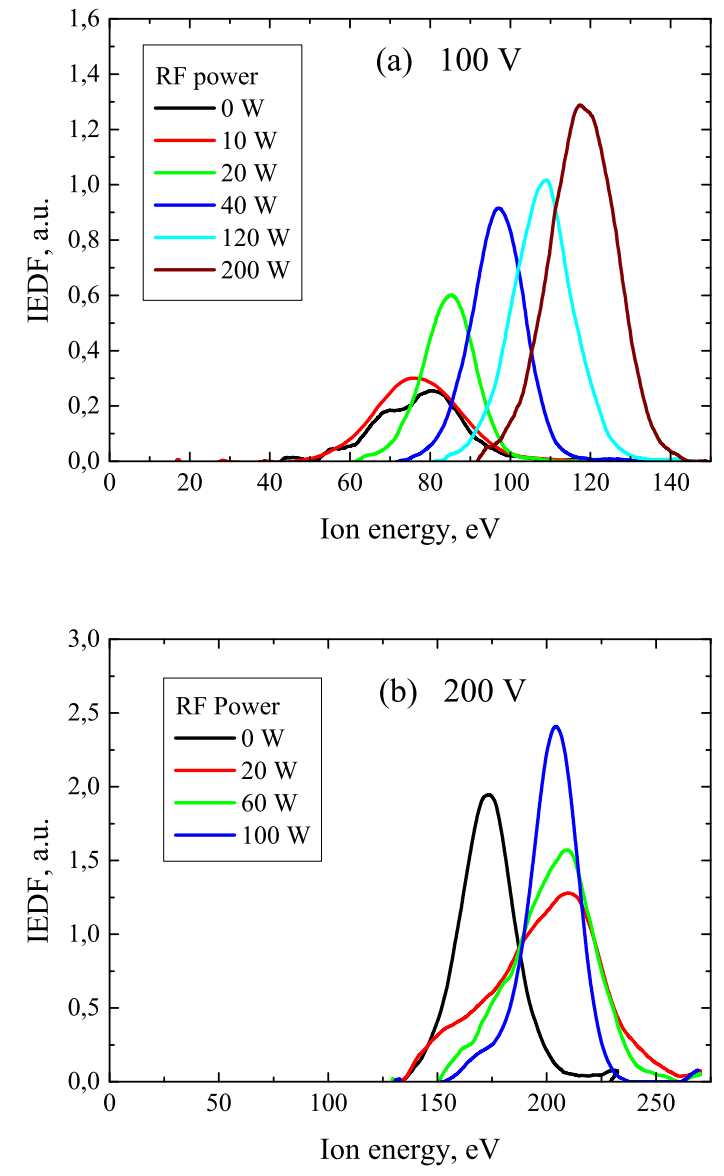

FIG. 14. IEDF for different values of the RF injected power and for two values of the discharge voltage, (a) $100 \mathrm{~V}$, and (b) $200 \mathrm{~V}$.

The mean ion speed can be obtained from the IEDFs as measured by the RPA:

$$
\langle v\rangle=\int v(\varepsilon) f(\varepsilon) d \varepsilon=\int \sqrt{\frac{2 \varepsilon}{m}} f(\varepsilon) d \varepsilon
$$

The mean cosinus, giving the component of the velocity directed along the thruster axis, can be obtained from the angular distribution measured by the Faraday probe:

$$
\langle\cos (\theta)\rangle=2 \pi R \int_{0}^{\pi / 2} \cos (\theta) f(\theta) \sin (\theta) d \theta
$$

And finally the mass rate can be simply calculated by transforming the ion current in A to a mass rate in $\mathrm{Kg} / \mathrm{s} \dot{m}=I_{i} / q \cdot m_{i}$ where $q$ is the elementary charge and $m_{i}$ the ion mass. The resulting thrust is shown in figure 17 as a function of the RF power for different values of the discharge voltage. There is an increase in thrust with RF power for $0 \mathrm{~V}, 50 \mathrm{~V}$ and $100 \mathrm{~V}$, while there is no clear trend at 150 and $200 \mathrm{~V}$. This shows that operating in double stage can be useful at low voltages, when single stage operation does not efficiently ionizes the gas. The thrust-to-power ratio is shown in figure 18 , taking into account
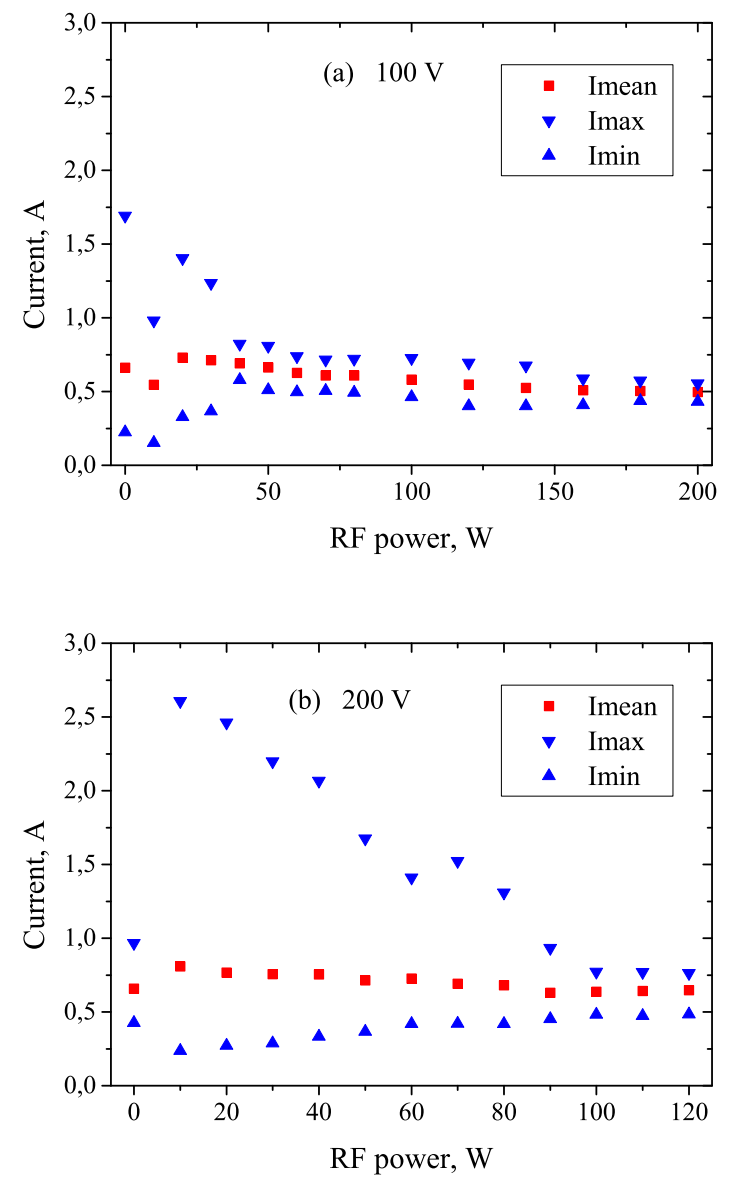

FIG. 15. Mean discharge current (squares) and maxima and minima of the current oscillations (up and down triangles) as a function of the RF power for two values of the discharge voltage, (a) $100 \mathrm{~V}$, and (b) $200 \mathrm{~V}$.

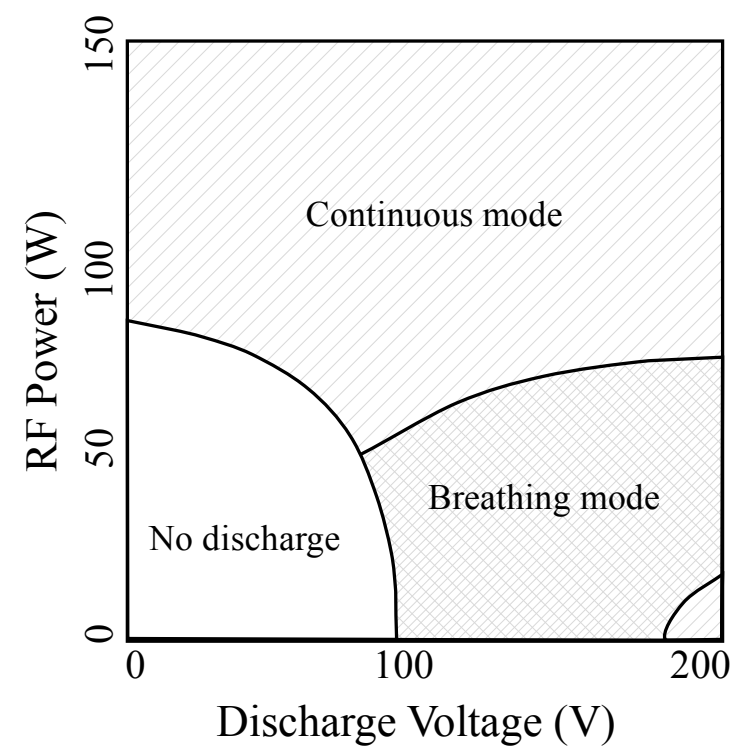

FIG. 16. Schematic diagram of the different regimes of ID-Hall. 
both DC and RF power. Here again we see the rapid decrease at high voltage, again indicating the regimes where the double stage may not be efficient. At $50 \mathrm{~V}$, the thrust-to-power, although lower than at higher voltages, remains fairly constant for all the power range, indicating a constant energy cost per ion as well as a fixed acceleration of each ion. At $100 \mathrm{~V}$, there is a combination of both effects: operation in single stage is possible at $100 \mathrm{~V}$ and has a higher $\mathrm{T} / \mathrm{P}$ ratio, which is higher than in double stage; however we are near the lower limit of single stage operation and the decrease of T/P as the RF power increases is not as steep as at higher voltages.

Figure 19 shows the efficiency of ID-Hall for different discharge voltages as a function of the total $(\mathrm{DC}+\mathrm{RF})$ power. When comparing the efficiency of double stage and single stage operations, one must take into account the fact that the efficiency for double stage operation is lower by a factor $\frac{P_{D C}}{P_{D C}+P_{R F}}$ than the single stage efficiency, where $P_{D C}=V I_{T}$ is the power associated with the discharge DC voltage and current, and $P_{R F}$ is the power absorbed in the ionization stage. The efficiency can be written as:

$$
\mu=\frac{1}{2} \frac{T^{2}}{\dot{m} P_{T}}=\frac{I_{i}}{I_{a}} \frac{I_{i}}{I_{T}} \frac{1 / 2 m v_{x}^{2}}{e\left(V+\frac{P_{d}}{I_{T}}\right)}
$$

In other words, assuming full ionization of the xenon flow, negligible electron current entering the channel with respect to the ion current, and a perfect ion beam at the energy corresponding to the applied voltage, the efficiency could be close to 1 in single stage operation but only $\frac{P_{D C}}{P_{D C}+P_{R F}}$ in double stage operation. For instance, for a double stage thruster with discharge voltage and current of $100 \mathrm{~V}$ and 0.5 A respectively and a power $P_{R F}=150 \mathrm{~W}$ absorbed in the ionization stage, the maximum efficiency that can be expected is $\eta_{\text {Max }}=0.25$. It can therefore be rather inefficient to operate under conditions where $P_{R F}$ is significantly larger than $P_{D C}$ (the same is true for gridded ion thrusters). This shows that the efficiency of a double stage operation will be generally lower than that of single stage operation. Optimization of the efficiency of double stage operation requires a good confinement of the ionization stage and a minimization of the electron current entering the thruster. The advantages of double stage thrusters may come from extended operating conditions not accessible to single stage thrusters (eg operation with alternative gases), from a better control of current oscillation and for an improved divergence of the ion beam.

\section{CONCLUSIONS}

A first prototype of the ID-HALL thruster has been built and tested at LAPLACE. The characterization techniques include measurement of the discharge current, Faraday probe measurements of the angular distribution of the ion plume and RPA measurements of the time averaged IEDF and also as a function of time whenever regular current oscillations were present. The characterization was performed with a fixed mass flow rate of $0.6 \mathrm{mg} / \mathrm{s}$ of xenon $(6 \mathrm{sccm})$.

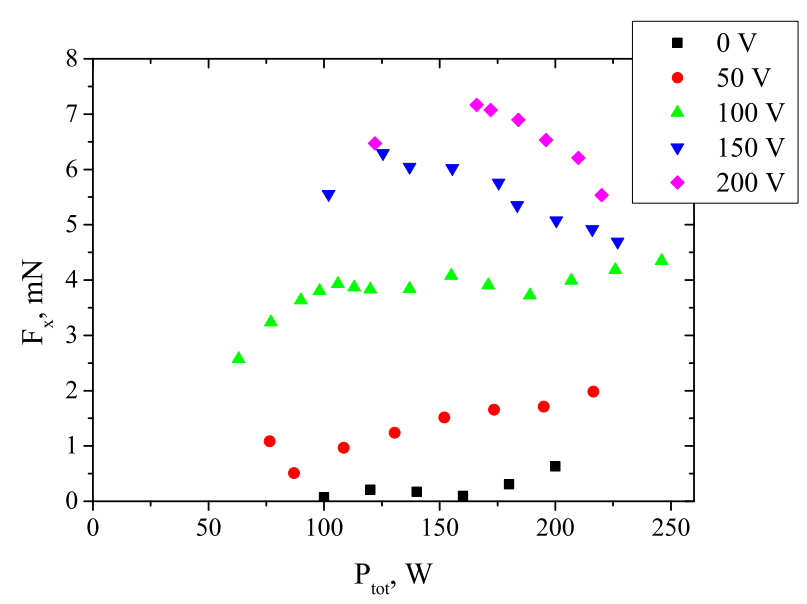

FIG. 17. Estimated thrust as a function of the total anode power (DC + coupled RF) for different fixed voltages.

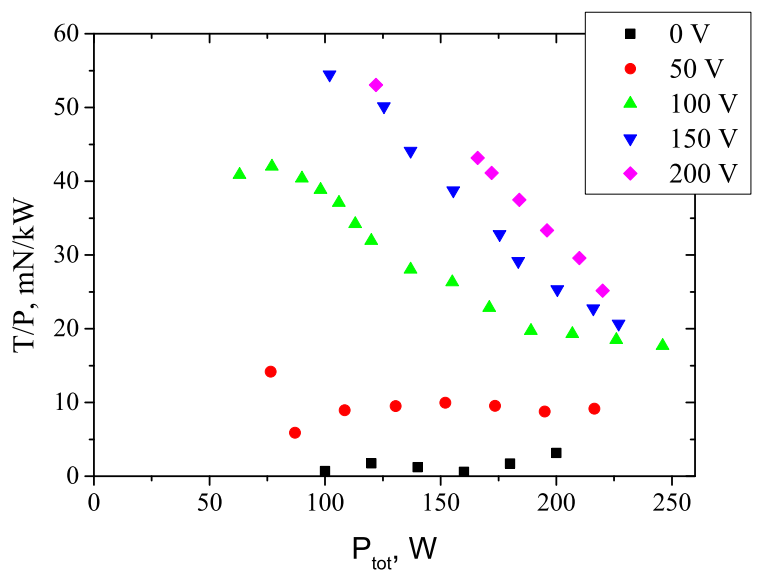

FIG. 18. Estimated thrust-to-power ratios as a function of the total anode power (DC + coupled RF) for different fixed voltages.

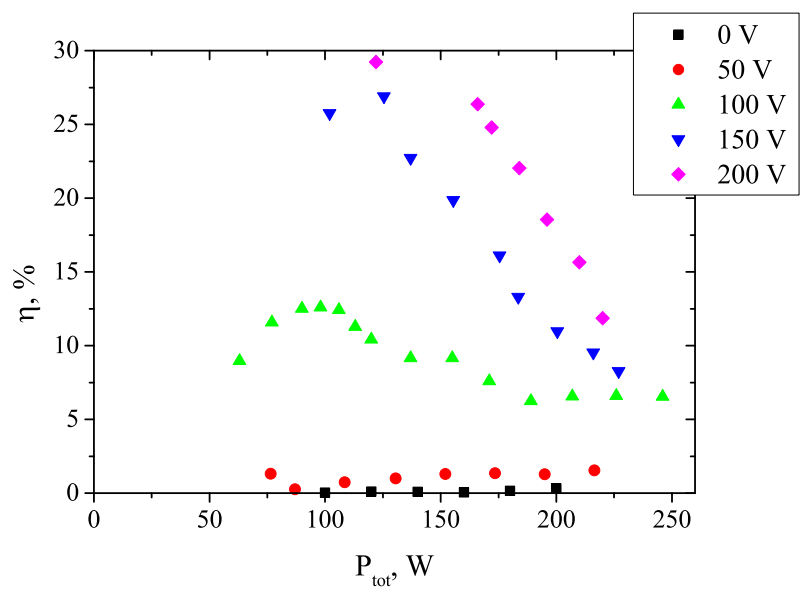

FIG. 19. Estimated anode efficiency as a function of the total anode power $(\mathrm{DC}+$ coupled RF) for different fixed voltages. 
In single stage operation, i.e. without power in the inductive RF plasma source, strong breathing mode oscillations are present below $190 \mathrm{~V}$ ("breathing mode") while only small amplitude random fluctuations are observed above $190 \mathrm{~V}$ ("continuous mode"). The with of the IEDF is larger in the breathing mode than in the continuous mode; in both cases the maximum of the IEDF was lower than the applied voltage. The reason is the presence of a second magnetic barrier in front of the anode, where a potential drop appears which does not accelerate the ions. This potential drop oscillates when breathing oscillations are present, leading to a corresponding energy shift of the instantaneous IEDFs and to a wider time-averaged IEDFs.

In double stage operation, with power in the inductive RF plasma source, it is possible to operate ID-Hall at voltages lower than the minimum voltage required for single stage operation (below $100 \mathrm{~V}$ ); in those conditions the ionization and acceleration are effectively decoupled, proving that ID-Hall can effectively operate as a true double stage Hall thruster. At intermediate voltages, between $100 \mathrm{~V}$ and $200 \mathrm{~V}$, the main effect of the double stage is the reduction of the discharge current without changing the total ion current; the electron current that crosses the magnetic barrier in the exhaust region therefore decreases. In single stage operation this region presents an overshoot of the total current (this feature is common to most single Hall thrusters), allegedly due to a larger anomalous or collisional electron transport; the decrease of the electron current entering the channel in double stage operation suggests that there is a significant change in electron transport across the magnetic barrier. For voltages higher than $200 \mathrm{~V}$ there is no significant difference between single and double stage operation.

The breathing mode oscillations that appear in single stage operation tend to disappear in double stage operation for high enough RF power. Plume divergence also decreases in the intermediate voltage range. Finally, the IEDF in double stage operation shows an increase of the ion energy with increasing RF power, even beyond the applied discharge voltage. This increase is caused by the large plasma potential inside the ionization chamber, due to the increasing electron temperature associated with the depletion of neutral atoms.

These results show the possibility of operating in double stage whenever single stage operation is not possible or inefficient, as in the case of low discharge voltages. It will be especially interesting to use propellant with lower atomic mass and/or less efficient ionization than xenon, because the same specific impulse can be obtained with lower voltage and supplementary ionization will be required in these conditions. Operation of ID-Hall with argon, krypton, and other gases will be explored in future work. Optimization of the magnetic field in the ionization chamber to increase confinement, as well as increasing the coupling efficiency of the ICP source will be also attempted. The results also show that the efficiency of the ionization source is not as good as expected and that the magnetic confinement of the plasma in the ionization chamber should be improved. Work is continuing toward optimization of the present magnetic configuration of ID-Hall and with a new design based on the magnetic shielding concept.

\section{ACKNOWLEDGMENTS}

This work was supported by the French Space Agency CNES and by the RTRA STAE foundation. A. Guglielmi benefits from a Ph.D. fellowship financed by the CNES and the Région Occitanie.

${ }^{1}$ Y. Peterson, Ph.D. thesis, Michigan State University (2004).

${ }^{2}$ A. I. Bugrova, A. Desyatskov, V. Kharchevnikov, A. Morozov, and M. Priol, in 29th International Electric Propulsion Conference, IEPC2005-146, Princeton, USA (2005).

${ }^{3}$ C. Boniface, G. J. Hagelaar, L. Garrigues, J. P. Boeuf, and M. Prioul, IEEE Transactions on Plasma Science 33, 522 (2005).

${ }^{4}$ L. Garrigues, C. Boniface, G. J. M. Hagelaar, and J. P. Boeuf, Physics of Plasmas 15, 113502 (2008).

${ }^{5}$ P. Y. Peterson, D. R. Massey, A. Shabshelowitz, R. Shastry, and R. Liang, in 32nd International Electric Propulsion Conference, IEPC-2011-269, Wiesbaden, Germany (2011).

${ }^{6} \mathrm{H}$. Kuwano, H. Nakashima, and H. Kuninaka, in Proceedings of the 24th International Symposium on Space Technology and Science, ISTS 2004, Miyazaki, Japan (2004).

${ }^{7}$ G. Matticari, L. Biagioni, E. Gengembre, G. Noci, M. Berti, E. Chesta, M. Capacci, and A. Severi, in 40th AIAA/ASME/SAE/ASEE Joint Propulsion Conference and Exhibit, AIAA 2004-3771, Fort Lauderdale, Florida (2004).

${ }^{8}$ H. Kuwano, A. Ohno, H. Kuninaka, and H. Nakashima, in 30th International Electric Propulsion Conference, IEPC-2007-085, Florence, Italy (2007).

${ }^{9}$ L. Dubois, F. Gaboriau, L. Liard, D. Harribey, C. Henaux, L. Garrigues, G. J. H. Hagelaar, S. Mazouffre, C. Boniface, and J. P. Boeuf, Physics of Plasmas 25, 093503 (2018).

${ }^{10}$ L. Dubois, F. Gaboriau, L. Liard, C. Boniface, and J. P. Boeuf, Physics of Plasmas 25, 093504 (2018).

${ }^{11}$ L. Dubois, F. Gaboriau, L. Liard, D. Harribey, C. Henaux, J. P. Boeuf, S. Mazouffre, and C. Boniface (2017).

${ }^{12} \mathrm{~A}$. Leufroy, T. Gilbert, and A. Bouchoule, in 31st International Electric Propulsion Conference, IEPC-2009-083, University of Michigan, USA (2009).

${ }^{13}$ R. Joussot, L. Grimaud, S. Mazouffre, C. Boniface, S. Oriol, and F. Masson, in The 5th Space Propulsion Conference, SP2016-3124900, Rome, Italy (2016).

${ }^{14}$ L. Dubois, Ph.D. thesis, Université Toulouse 3 - Paul Sabatier (2018).

${ }^{15}$ F. Diop, T. Gibert, and A. Bouchoule, Physics of Plasmas 26, 063508 (2019).

${ }^{16}$ Y. Raitses, T. Moeller, and J. Szabo, in 30th International Electric Propulsion Conference, IEPC-2007-334, Florence, Italy (2007).

${ }^{17} \mathrm{D}$. Brown and A. Gallimore.

${ }^{18}$ N. Gascon, M. Dudeck, and S. Barral, Physics of Plasmas 10, 4123 (2003).

19 "See https://www.hallis-model.com for hallis software,".

${ }^{20}$ G. J. Hagelaar, J. Bareilles, L. Garrigues, and J. P. Boeuf, Journal of Applied Physics 91, 5592-5598 (2002).

${ }^{21}$ V. A. Godyak, Plasma Sources Science and Technology 20, 025004 (2011). 